\title{
VHDL Implementation of DSDV Ad-Hoc Routing Protocol
}

\author{
Priya Singh ${ }^{1}$, Ankita Rastogi ${ }^{2}$ \\ ${ }^{1,2}$ Student,M.Tech. (VLSI Design), Department of Electronics, Banasthali University, Rajasthan, India
}

\begin{abstract}
An Ad-Hoc network deals with the collection of mobile nodes without any centralized structure. This can be well suited for environment where changes are frequent and establishment of infrastructure is not very cost effective. In short it can turn the dream of 'anytime anywhere' into reality[1]. Here we deal with the VHDL implementation of DSDV(destination sequenced distance vector) routing protocol to fulfill these requirements of Ad-hoc network more effectively.

Keywords: Adhoc, DSDV,FPGA,VHDL, Routing
\end{abstract}

\section{Introduction}

For the Ad-hoc network we need to deal with various issues of routing protocol, security etc. For handling the changes in Ad-hoc network efficiently some protocols had been developed as a solution known as routing protocols. It is mainly categorized in two types: 1) Link State Routing Protocol 2) Distance Vector Routing Protocol. Based on these divisions various protocols had been developed so far, one of these is DSDV. DSDV is the proactive routing protocol in which data packets are exchanged between various nodes or stations of the network. It is introduced mainly from conventional Routing information Protocol(RIP) and is using advanced version of Bellman Ford algorithm. It includes sequence number to each route table entry with metric number, destination Id, Source Id and number of hops required for completion of route [2]. Based on these table entries route is maintained and erased. By the use of sequence number the mobile nodes can distinguish between the stale route information and thus preventing the routing of negative loops.

\section{VLSI Architecture of DSDV}

The VLSI architecture of DSDV protocol [3] has been depicted in Fig.1. It consists of Data Link in buffer to store the input control information to be transmitted from nodes and received by node in tabular format. Data Link out buffer is use to store output control information that is forwarded to neighbours (nodes). RIM (route information memory) stores the information necessary to identify the next hop address for optimized route.

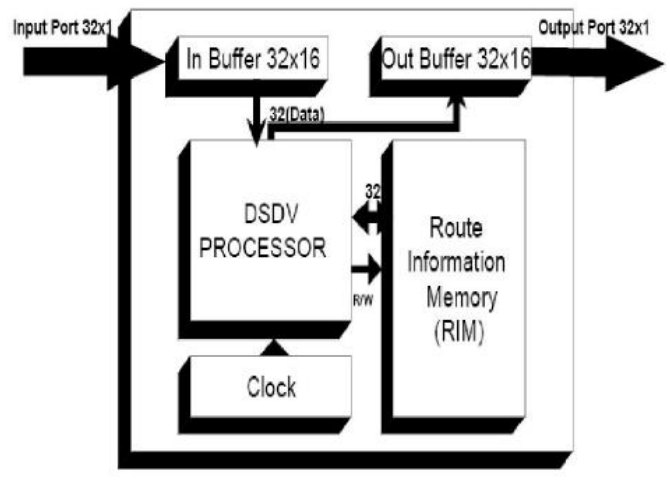

Figure1. The implemented architecture of MANET node[1]

The FSM of DSDV control unit had been depicted in Fig.2. It processes the three states with the help of three processors. It has four states 1) Idle state 2)Table Transmission (periodic update) 3) Receive Input 4) Checking Stale Node.[5,7] 


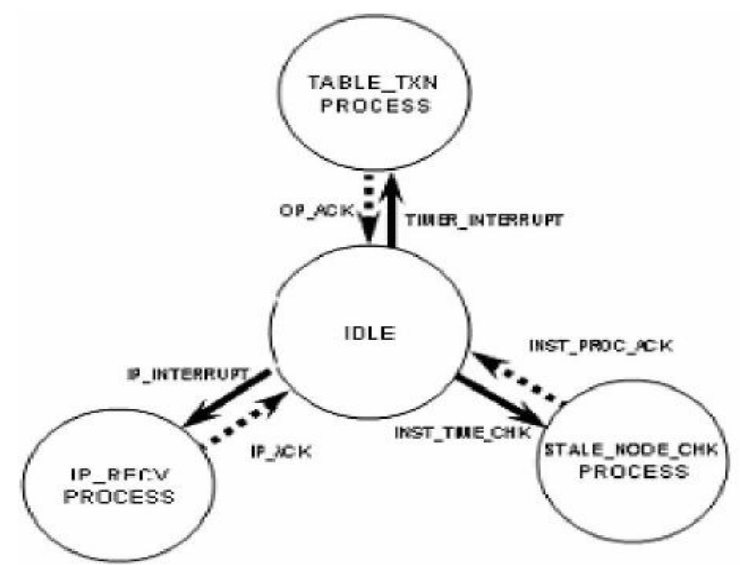

Figure2. Sequence of three main functions in DSDV processor [4]

\section{FPGA Implementation}

The shown architecture of DSDV control unit is implemented using VHDL coding with simulation performed on ModelSim Simulator and synthesis report had been obtained by Leonardo Spectrum of Mentor Graphics.

\subsection{Simulation Report}

Simulation waveform of DSDV control unit has been depicted in Fig.3.

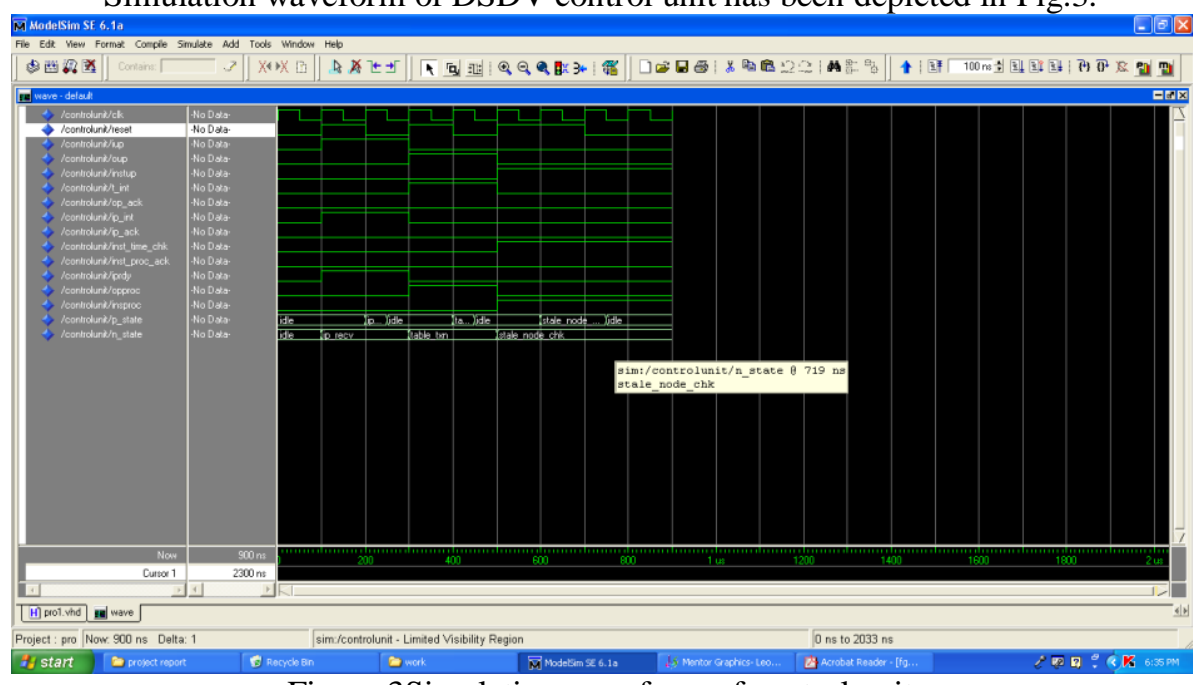

Figure 3Simulation waveform of control unit

\subsection{Synthesis Report}

The synthesis performed on Leonardo Spectrum gives the following report

\subsubsection{Synthesis Report}

Cell: controlunit View: behavioral Library: work

Cell: controlunit View: behavioral Library: work

Cell Library References Total Area

Cell Library References Total Area

AN3T0 scl05u 2 x $6 \quad 12$ gates

AN4T0 scl05u 2 x $8 \quad 16$ gates

FD1B0 scl05u $2 \times \quad 9 \quad 17$ gates

IV1N0 scl05u $3 x \quad 3 \quad 9$ gates

LD1A0 scl05u $5 \mathrm{x} \quad 7 \quad 33$ gates

MX2L0 scl05u $1 \times \quad 6 \quad 6$ gates

ND2N0 scl05u $1 x \quad 5 \quad 5$ gates

NR2R1 scl05u $1 x \quad 5 \quad 5$ gates

NR3R0 $\quad$ scl05u $1 x \quad 6 \quad 6$ gates

OA1R0 scl05u 1 x $6 \quad 6$ gates 
OAI3N0 $\quad$ scl05u 1 x $\quad 8 \quad 8$ gates

OAOI0 scl05u $1 \mathrm{x} \quad 8 \quad 8$ gates

Number of ports : $\quad 14$

Number of nets : $\quad 33$

Number of instances : $\quad 21$

Number of references to this view : 0

Total accumulated area :

Number of gates :

Number of accumulated instances : 21

Info, Command 'report_area' finished successfully

->report_delay -num_paths 1 -critical_paths -clock_frequency

Using default wire table: SCL_CORE_4K

Clock Frequency Report

Clock : Frequency

$$
\text { clk } \quad: 461.8 \mathrm{MHz}
$$

Critical Path Report

Critical path \#1, (unconstrained path)

Critical path \#1, (unconstrained path)

\section{NAME GATE ARRIVAL LOAD}

clock information not specified

delay thru clock network

reg_p_state $(0) / Q$

FD1B0

0.00 (ideal)

ix $332 / \mathrm{X}$

NR2R1

$0.000 .48 \mathrm{dn}$

0.26

ix350/X

OAOIO

0.521 .00 up

0.34

ix69/X

OAI3NO

0.351 .29 up

0.06

ix 337/X

IV1N0

lat_iprdy/G

LD1A0

$0.421 .71 \mathrm{dn}$

data arrival time

0.402 .11 up

0.28

data required time

$0.002 .11 \mathrm{up}$

0.00

data required timenot specified data arrival time

2.11

not specified

unconstrained path

RTL synthesis, circuit representation and block diagram view of DSDV Control unit has been depicted in Fig.4,Fig.5 andFig. 6 respectively.

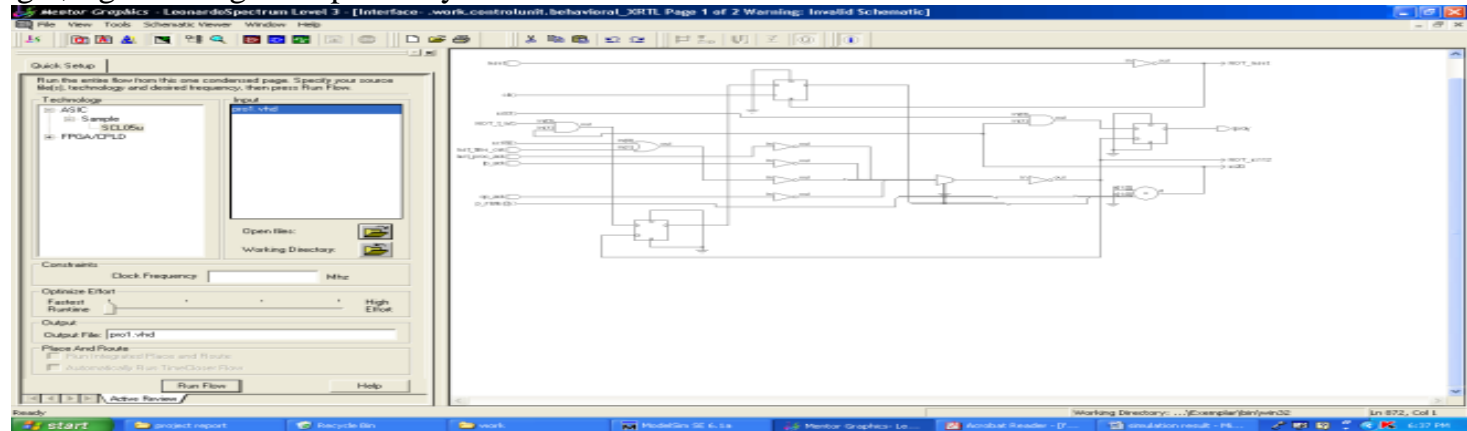

Figure 4 RTL schematic of control unit 


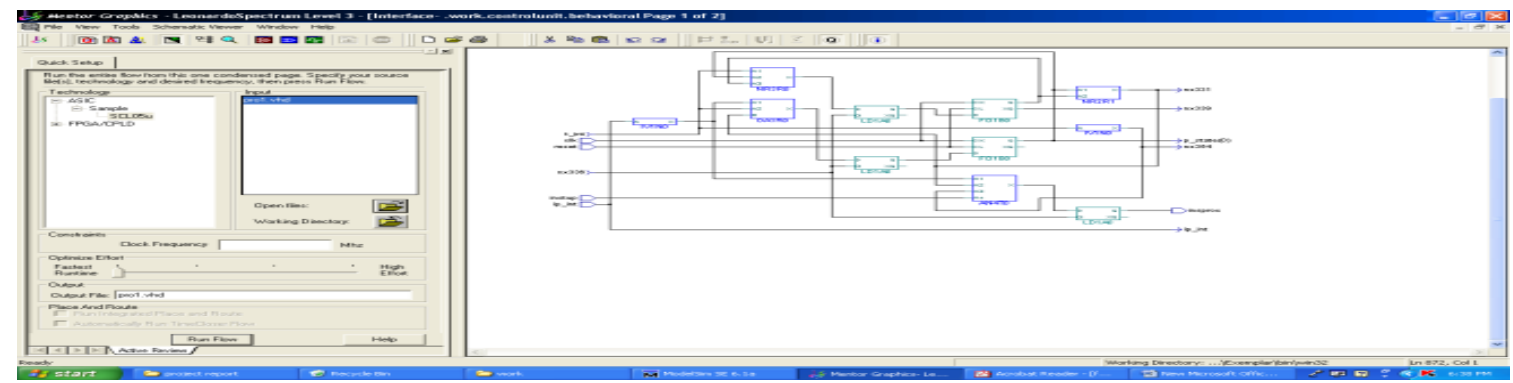

Figure 5 Technological view of control unit

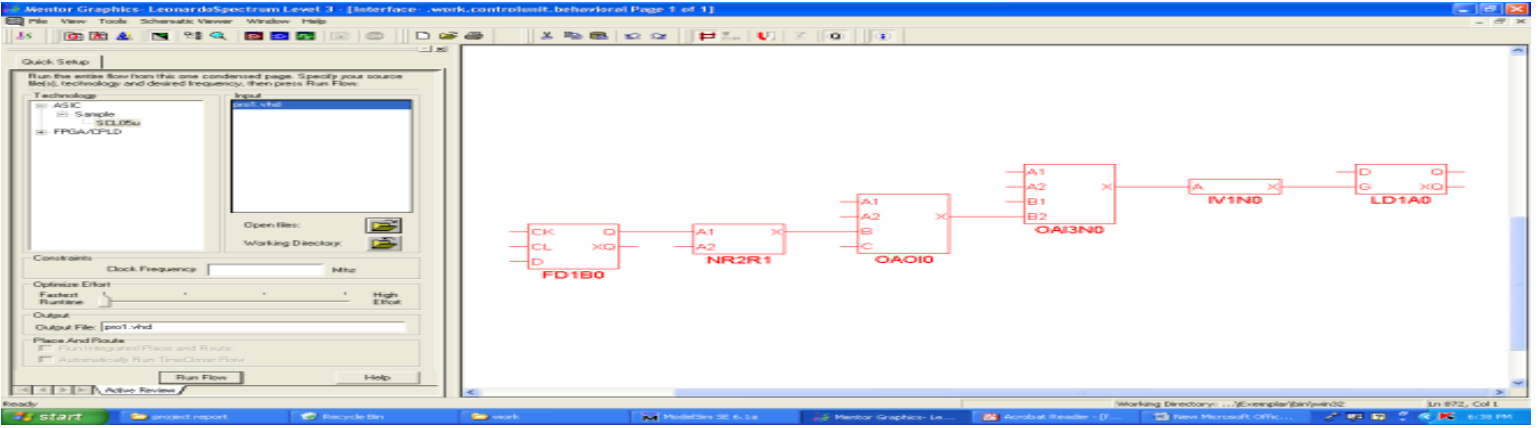

Figure 6 Critical path of control unit

MHz.

This report had obtained a much optimized arrival time of $\mathbf{2 . 1 1}$ nsec only and clock frequency of $\mathbf{4 6 1 . 8}$

\section{Conclusion}

From the results obtained from synthesis we can conclude that FPGA implementationof DSDV control unit can give the operating frequency in $\mathrm{MHz}$ and arrival time in nsecs, which is much optimized then its counterpart implementationtechnique.

\section{Future Scope}

The proposed architecture of control unit of DSDV gives reduced call setup time and quick handling of dynamic topology under huge traffic, thus improved speed and efficiency of router can be obtained. In future more optimized concept can be introduced by using reconfigurable protocol which can switch according to the requirement and condition of environment. This future advancement can provide a better clock frequency and arrival time by using FPGA implementation instead of software implementation such as using NS2.

\section{Acknowledgement}

We are highly thankful to our university Banasthali Vidyapith, from where we had got the great opportunity for writing a research paper. We also obliged to the faculty of Department of Electronics who helped us in carrying out this work.

\section{References}

[1]. P. Ramamoorthy, A. Shanmugametal, 'Performance Analysis of Congestion Aware Distance Vector Routing Protocol in MANET', International Journal of Systemics, Cybernetics and Informatics, pp-78 to 80, January 2006.

[2]. J. Broch, D. Maltz, D. Johnson, Y.C.Hu, and J. Jetcheva, 'A Performance Comparison of Multi-hop Wireless Ad-hoc Network Routing Protocols', Proc. Of Mobicom 1998, pp.85-97.

[3]. M. Ramakrishnan, Dr. S. Shanmugavel, 'New Approaches to Routing Techniques of MANET Node for Optimal Network Performance', IJCSNS International Journal of Computer Science and Network Security, VOL.8 No.11, November 2008.

[4]. M. Ramakrishnan, Dr. S. Shanmugavel, 'FPGA Implementation of DSDV Based Router In Mobile Ad-hoc Network', IEEE, ICIIS, August 2006, Sri Lanka.

[5]. Charles E. Perkins and Pravin Bhagwat, 'Highly Dynamic Destination Sequenced Distance Vector Routing for Mobile Computers' In Proceedings of the SIGCOMM '94 Conference pages 234-244, August 1994.

[6]. H. Wang, Veeraraghavan and R. Karri, 'A Hardware Implementation of a Signaling Protocol', in Proc. Of Opticomm, July, 29Aug, 2002, Boston ,M.A.

[7]. M. Ramakrishnan, Dr. S. Shanmugavel, 'Dynamic Reconfigurable Generic Router Architecture in MANET', in NCMEC06 @ SRM Institute of Science and Technology ,Chennai. pp 114-116.

[8]. C. Perkins , E. Belding Royer, S. Das, Network Working Group, 'Ad-hoc On Demand Distance Vector Routing', RFC 3561, July 2003.

Books

[9]. T. S. Rappaport, 'Wireless Communications-Principles and Practice', (Pearson Edition, 2003). 
[10]. [2] Douglas L. Perry, 'VHDL Programming By Example', (Tata McGraw-Hill, 2002).

[11]. [3] Charles E. Perkins, 'Ad-Hoc Networking', (Addition Wesley Publications , First Edition, 2000).

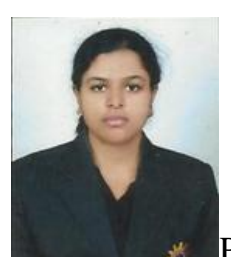

Priya Singh received her B.Tech. degree from RGTU Bhopal, M.P., India in 2012 and pursuing M.Tech. from Banasthali University.

Ankita Rastogi received her B.Tech. degree from UPTU Lucknow, U.P., India in 2011 and pursuing M.Tech. from Banasthali University. 